\section{To the membership of the Canadian Thoracic Society}

Dear Colleagues;

On Friday, February 4, 2005, there was a successful meeting between the Canadian Lung Association (CLA) and Canadian Thoracic Society (CTS) Executives. We discussed the relationship between the two organizations and achieved an improved understanding of each other's situation and needs. I believe the foundation has been set for us to move forward with achieving our goals in a positive and mutually beneficial relationship.

In retrospect, it is evident that the leadership of the CLA did not foresee the far-reaching implications of the dismissal of Valoree McKay, who had provided administrative support to our society. It is clear that the negative consequences to our organization were largely unanticipated and were definitely not intended. It was agreed that in the future, our Executive would be informed of potential changes in the staff that provide administrative support to the CTS.

On the matter of administrative support for our organization, we are very fortunate to have the opportunity of working with Ms Debbie Smith, who brings a wealth of relevant experience to this role. She joined us in early December and has fulfilled her responsibilities admirably. I have every confidence that Debbie will provide expert support to the CTS. Please join me in welcoming Debbie Smith to her new position.

Among our key priorities at present are the organization of the National Grant Review process and planning for the CTS elements of the American College of Chest Physicians' 2005 meeting, which will be held in Montreal. The committee chairs responsible for these activities have been very active, and Debbie Smith will work with them to assist in making sure that these key CTS programs continue to be successful. We are also very pleased to participate in the recruitment of a new staff member who will be responsible for the research agenda. We have struggled for some years to achieve a national profile for the respiratory community and we believe that the recruitment of a staff member with primary responsibility for the research agenda will aid this effort significantly.

With this reorganization, the CTS has the potential to refocus its activities and develop new initiatives. We appreciate the frank and transparent discussions that we have had with the CLA Executive and we look forward to improved relations between the organizations. Such a relationship will enable us both to achieve our shared goal to improve respiratory health in Canada.

Yours faithfully,

P Gerard Cox MB FRCPC President, Canadian Thoracic Society

\section{Lettre aux membres de la Société canadienne de thoracologie}

Chère consœur,

Cher confrère,

Le vendredi 4 février 2005 a eu lieu une réunion fructueuse des comités directeurs de l'Association pulmonaire du Canada (APC) et de la Société canadienne de thoracologie (SCT). Nous avons discuté des liens entre les deux organismes et sommes parvenus à une meilleure compréhension de la situation et des besoins de chacun. Je pense que les assises sont posées pour aller de l'avant et atteindre nos objectifs dans le cadre d'une relation positive et mutuellement avantageuse.

En rétrospective, il est évident que la direction de l'APC n'avait pas prévu les lourdes conséquences du licenciement de Valoree McKay, qui assurait le soutien administratif de notre société. Il est clair que la plupart des conséquences négatives pour notre société n'avaient pas été prévues et qu'elles n'étaient absolument pas voulues. Il a été convenu qu'à l'avenir, notre comité directeur serait informé des modifications potentielles au personnel qui assure le soutien administratif de la SCT.

À cet égard, nous avons la chance de travailler avec madame Debbie Smith, qui possède une expérience vaste et pertinente du soutien administratif rôle. Arrivée au début de décembre, elle assume admirablement ses responsabilités. Je suis persuadé que Debbie offrira un soutien expert à la SCT. Souhaitez-lui la bienvenue avec moi dans son nouveau poste.

Parmi nos priorités, soulignons l'organisation du processus national de révision des subventions et la planification de la partie du congrès 2005 de l'American College of Chest Physicians relevant de la SCT. Ce congrès aura lieu à Montréal. Les présidents des comités responsables de ces activités sont très actifs, et Debbie Smith travaillera avec eux pour que ces programmes essentiels de la SCT continuent de porter fruit. Nous sommes également très heureux de participer au recrutement d'un nouvel employé qui sera responsable du programme de recherche. Nous cherchons depuis plusieurs années à nous donner un profil national au sein du milieu pulmonaire, et nous sommes d'avis que l'embauche d'une telle personne contribuera énormément à cet effort.

Grâce à cette réorganisation, la SCT a le potentiel de réorienter ses activités et d'élaborer de nouveaux projets. Nous sommes satisfaits des discussions franches et transparentes tenues avec le comité directeur de l'APC, et nous comptons sur de meilleures relations entre les deux organismes. Cette relation nous permettra tant de réaliser nos objectifs que d'améliorer la santé respiratoire au Canada.

Je vous prie d'agréer, chère consœur, cher confrère, mes sincères salutations.

$P$ Gerard Cox MB FRCPC Président, Société canadienne de thoracologie 


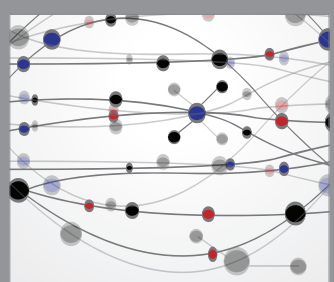

The Scientific World Journal
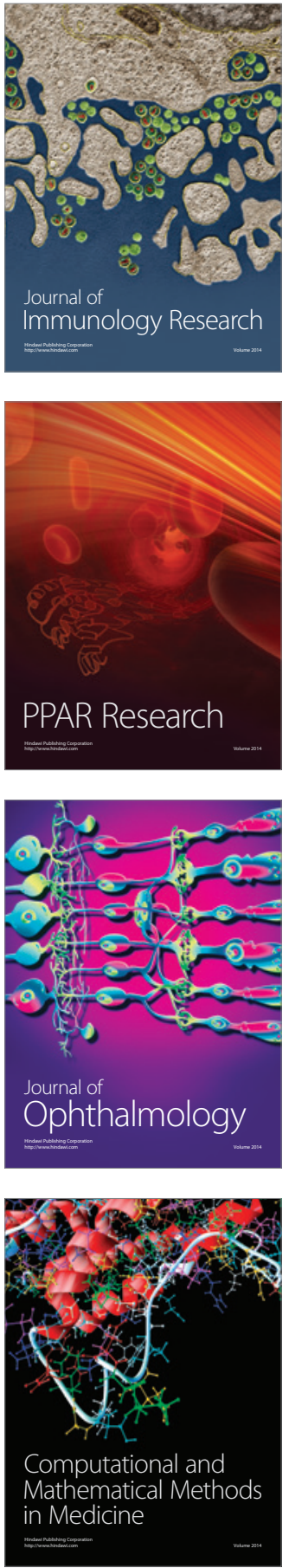

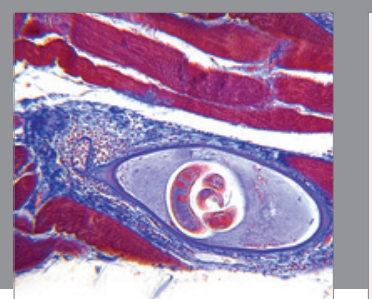

Gastroenterology Research and Practice

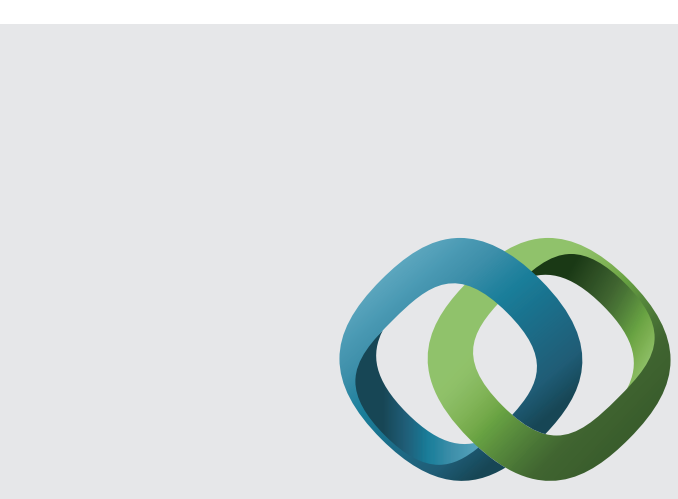

\section{Hindawi}

Submit your manuscripts at

http://www.hindawi.com
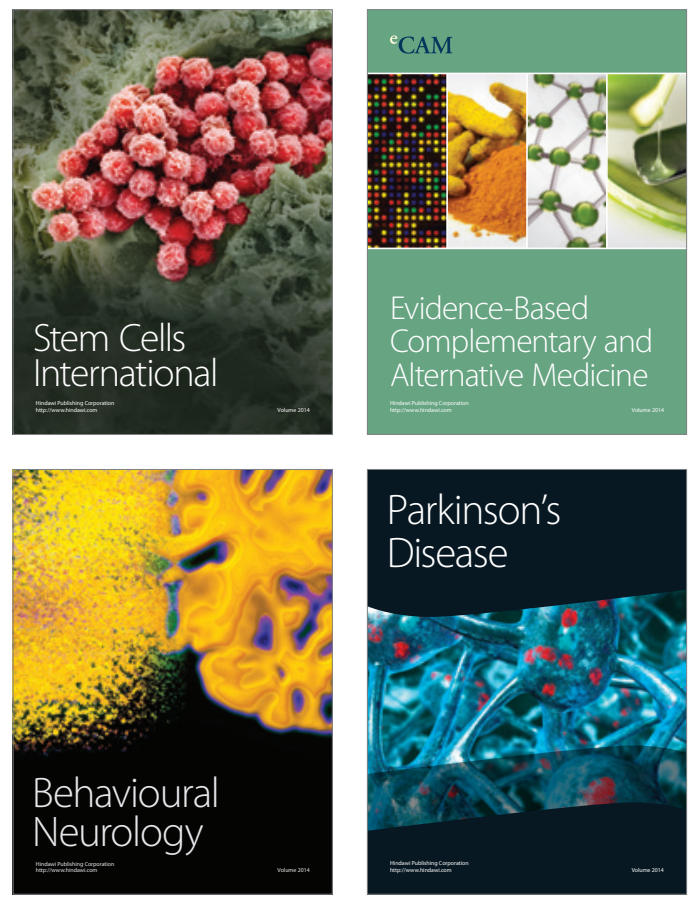
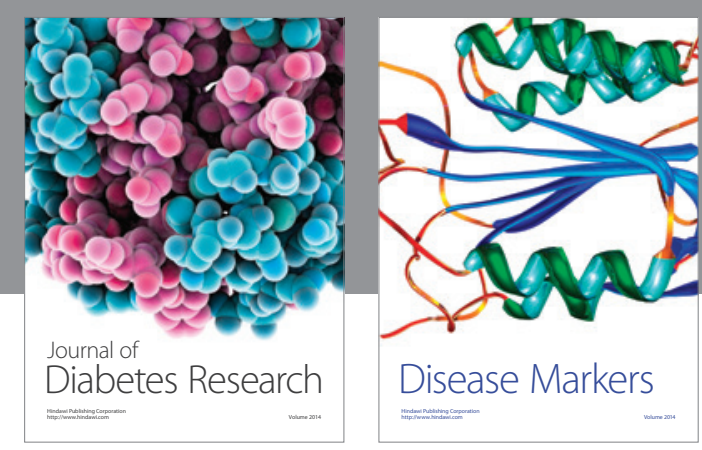

Disease Markers
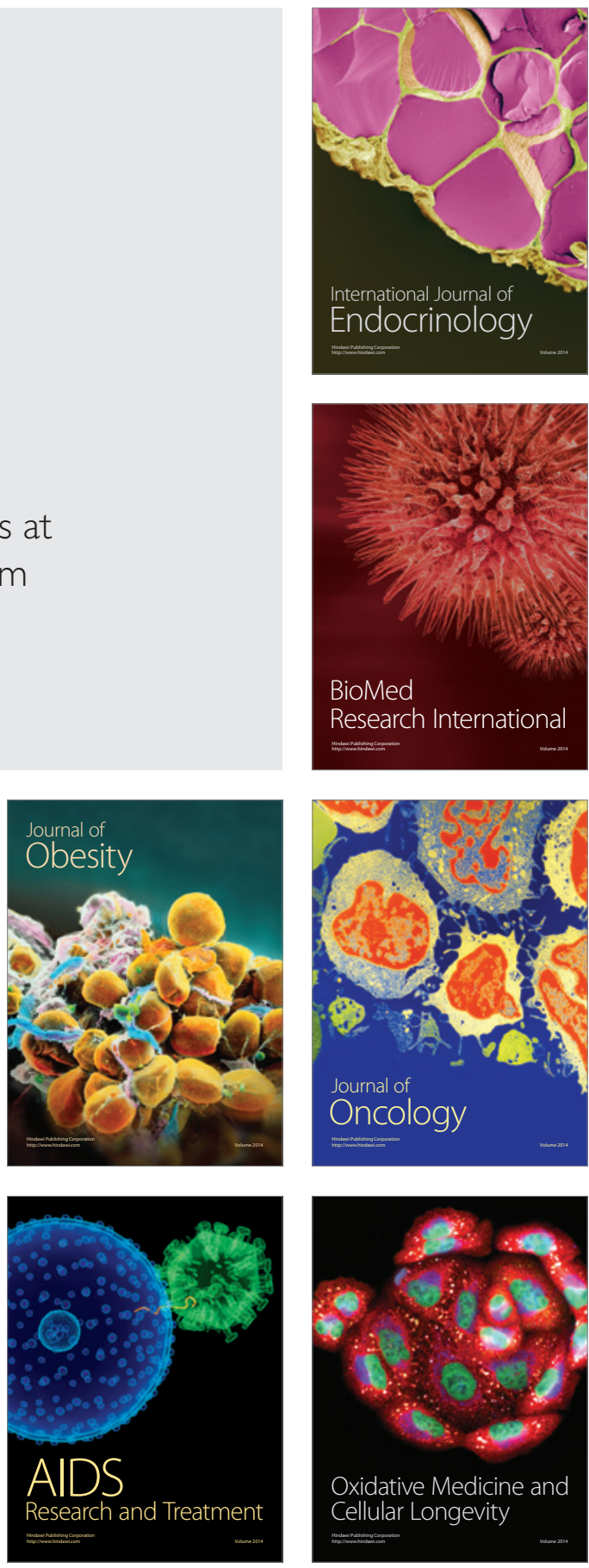